$4 \mathrm{mmol} / \mathrm{L}$. The lactate level is most frequently measured in the arterial blood gas (ABG). Aim of this study is to evaluate the agreement of venous blood gas (VBG) lactate compared to ABG lactate.

Method Prospectively collected data from twenty patients were included in this study. All patients were admitted to the emergency department. ABG and three types of VBG samples were collected from each patient. To compare the VBG samples, processing was done in three different ways; VBG1 was held steady and analysed within $5 \mathrm{~min}, \mathrm{VBG} 2$ was tilted for $5 \mathrm{~min}$ and analysed within $7 \mathrm{~min}, \mathrm{VBG} 3$ was held steady and analysed after $15 \mathrm{~min}$. ABG and VBG samples were compared using Bland-Altman plot.

Results The Bland-Altman plot showed narrow 95\% limits of agreement and average differences in lactate of $-0,24,-0,25$ and $-0,33$ between $A B G$ and VBG1-3, respectively.

Conclusion Venous blood lactate is a valid parameter when measuring the level of lactate. However larger studies are needed to further evaluate the reliability regarding patients with severe hyperlactataemia.

Conflict of interest None declared

Funding This study did not receive external funding.

\section{OUT-OF-HOSPITAL TREATMENT OF ACUTE FOREIGN BODY AIRWAY OBSTRUCTION IN ADULTS: A STRUCTURED LITERATURE REVIEW}

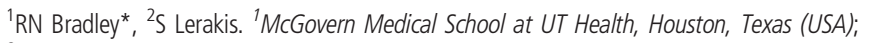
${ }^{2}$ Emory University, Atlanta, Georgia (USA)

\subsection{6/10.1136/bmjopen-2018-EMS.31}

Aim There were 4700 deaths due to choking in the United States in 2012. This study was a structured literature review with the objective of determining whether in adults, either conscious or unconscious, with acute foreign body airway obstructions, if any specific resuscitation technique, compared to other techniques, leads to different outcomes.

Method We developed an a priori search definition, and searched PubMed, Google, and OneSearch@IU. We sought additional articles by reviewing the reference lists of articles that we included. We included articles if they addressed original research of treatment of foreign body airway obstruction in adults. We excluded articles if the skill was not applicable to an out-of-hospital rescuer or if there was no abstract available in English. For those articles that met our criteria, two investigators independently collected the results and assessed the quality of the evidence.

Results We identified 534 articles for screening and performed full-text reviews on 64 . We included 44 articles in our qualitative synthesis. We found one fair quality study that supported the use of abdominal thrusts, two fair quality studies that supported back blows, and one fair quality study that supported chest thrusts.

Conclusion Rescuers attempting to resolve a complete foreign body airway obstruction in a conscious adult should provide back blows and either abdominal and/or chest thrusts to the victim. Rescuers attempting to resolve a complete foreign body airway obstruction in an unconscious adult should provide CPR and use a finger sweep if a foreign body is seen in the mouth.

Conflict of interest RNB and SL are both employed by their respective medical schools. Some travel expenses are reimbursed as SL is a member and RNB is the chair of the Resuscitation Subcouncil of the American Red Cross Scientific Advisory Council.

Funding None

\section{DERIVATION OF A NOVEL INPATIENT MORTALITY PREDICTION MODEL FOR EMERGENCY DEPARTMENT PATIENTS IN SINGAPORE}

${ }^{1}$ Stella Xinzi Wu*, ${ }^{1,2}$ Nan Liu, 1,3Marcus Eng Hock Ong. 'Duke-NUS Medical School, National University of Singapore, Singapore; ${ }^{2}$ Health Services Research Centre, Singapore Health Services, Singapore; ${ }^{3}$ Department of Emergency Medicine, Singapore General Hospital, Singapore

\subsection{6/10.1136/bmjopen-2018-EMS.32}

Aim Inpatient mortality is an indicator of hospital performance and patient care. In this retrospective cohort study, we aimed to develop and validate an inpatient mortality model for use during ED consultation to efficiently risk stratify patients for better care and resource allocation.

Method Data was extracted from the Electronic Health Records (unified patient care data) of Singapore General Hospital in the year of 2014. Patients admitted through the ED were included and patients $<21$ were excluded. Variables, such as demographics, comorbidities, socioeconomic status and laboratory tests, were selected through literature review and clinician judgement before analysis with univariable and multivariable logistic regression. The model was assessed with receiver operating characteristic area under the curve.

Results Among the 35699 patients admitted from ED in 2014, 690 died in-hospital. Univariate-analysis showed males, lower socioeconomic status, multiple comorbidities and increased acuity of illness as significant variables contributing to inpatient mortality. The final model included gender, use of medifund (financial aid), Charlson Comorbidity Index (CCI), albumin, creatinine, white blood cell counts, and number of ED visits within the past 1 year. This model (AUC 0.840) performed the best compared to other scores, including just using CCI, age, gender and principal diagnosis (AUC 0.723).

Conclusion A novel model for predicting inpatient mortality could effectively risk stratify patients early in the ED. This model may have future applications to improve management and disposition.

Conflict of interest None

Funding None

\section{INTERACTION EFFECTS BETWEEN TARGETED TEMPERATURE MANAGEMENT AND HYPERTENSION ON SURVIVAL OUTCOMES AFTER OUT-OF-HOSPITAL CARDIAC ARREST}

Jung Eujene*. Chonnam National University Hospital Clinical Fellow

\subsection{6/10.1136/bmjopen-2018-EMS.33}

Aim Targeted temperature management (TTM) currently represents the most efficacious treatment to improve neurological recovery in comatose patients who have return of spontaneous circulation (ROSC) after out-of-hospital cardiac arrest (OHCA). The effect of TTM with HTN have not been reported. This study aimed to investigate whether the effect of TTM on neurological recovery after OHCA differed between patients with or without HTN. 